\title{
A novel body circumferences-based estimation of percentage body fat
}

\author{
Yair Lahav ${ }^{1}$, Yoram Epstein ${ }^{1,2}$, Ron Kedem ${ }^{1}$ and Haggai Schermann ${ }^{1,3 *}$ \\ ${ }^{1}$ Sheba Medical Center, Heller Institute of Medical Research, Tel Hashomer, Ramat Gan, Israel, 52621 \\ ${ }^{2}$ Sackler Faculty of Medicine, Tel Aviv University, Tel Aviv, Israel, 62431 \\ ${ }^{3}$ Division of Orthopedic Surgery, Tel Aviv Sourasky Medical Center affiliated with Tel Aviv University, Tel Aviv, Israel, 62431 \\ (Submitted 13 June 2017 - Final revision received 5 January 2018 - Accepted 8 January 2018)
}

\begin{abstract}
Anthropometric measures of body composition are often used for rapid and cost-effective estimation of percentage body fat (\%BF) in field research, serial measurements and screening. Our aim was to develop a validated estimate of \%BF for the general population, based on simple body circumferences measures. The study cohort consisted of two consecutive samples of health club members, designated as 'development' ( $n$ 476, 61\% men, 39\% women) and 'validation' ( $n$ 224, 50\% men, 50\% women) groups. All subjects underwent anthropometric measurements as part of their registration to a health club. Dual-energy X-ray absorptiometry (DEXA) scan was used as the 'gold standard' estimate of $\% \mathrm{BF}$. Linear regressions where used to construct the predictive equation $\left(\% \mathrm{BF}_{\mathrm{cal}}\right)$. Bland-Altman statistics, Lin concordance coefficients and percentage of subjects falling within $5 \%$ of \%BF estimate by DEXA were used to evaluate accuracy and precision of the equation. The variance inflation factor was used to check multicollinearity. Two distinct equations were developed for men and women: \% $\mathrm{BF}_{\mathrm{cal}}(\mathrm{men})=10 \cdot 1-0 \cdot 239 H+0 \cdot 8 A-0 \cdot 5 N ; \% \mathrm{BF}_{\mathrm{cal}}($ women $)=19 \cdot 2-0 \cdot 239 H+0 \cdot 8 A-0 \cdot 5 N$ ( $H$, height; $A$, abdomen; $N$, neck, all in cm). BlandAltman differences were randomly distributed and showed no fixed bias. Lin concordance coefficients of \%BF $\mathrm{Fal}_{\text {cal }}$ were 0.89 in men and 0.86 in women. About $79.5 \%$ of $\% B F$ predictions in both sexes were within $\pm 5 \%$ of the DEXA value. The Durnin-Womersley skinfolds equation was less accurate in our study group for prediction of $\% \mathrm{BF}$ than $\% \mathrm{BF}_{\mathrm{cal}}$. We conclude that $\% \mathrm{BF}_{\mathrm{cal}}$ offers the advantage of obtaining a reliable estimate of \%BF from simple measurements that require no sophisticated tools and only a minimal prior training and experience.
\end{abstract}

Key words: Percentage body fat: Anthropometry: Circumference: Skinfolds: Dual-energy X-ray absorptiometry

Anthropometry-based measurement of body composition offers the advantage of a simple and cheap estimation of an important parameter, which is associated with major health outcomes ${ }^{(1)}$. A valid approach can be effectively applied in field research, large cohort surveys, serial assessments and screening. This is in contrast to laboratory and imaging estimates of body composition that are usually costly and technically challenging ${ }^{(2-4)}$. Noteworthy, laboratory and imaging techniques are most valuable as 'gold standards' for validation of field measures and as the diagnostic modality for subjects with medical conditions or unusual complexity. Of these, dual-energy X-ray absorptiometry (DEXA) is universally accepted as the 'gold standard', due to its reliability and applicability ${ }^{(5)}$.

BMI is an example of a field measure that became widely used due to its simplicity. However, BMI correlates poorly with the body fat component estimation by $\operatorname{DEXA}^{(6,7)}$, tends to overestimate obesity in people with relatively high lean body mass (LBM), and to underestimate it in people with lower LBM. Over the years, various attempts have been made to construct equations for estimating percentage body fat (\%BF) based on skinfold thickness measurements ${ }^{(8-10)}$. In general, the skinfolds measurement method is advantageous for quantification of regional adiposity, which by itself is an independent predictor of morbidity at a given level of whole-body adiposity ${ }^{(11)}$. However, \%BF by skinfold measurements suffers a low reproducibility and high inter-observer agreement ${ }^{(12)}$. In this regard, predictive equations that are based on body circumferences are a more reliable method for estimating total adiposity. Indeed, waist circumference:height ratio is now the most preferred anthropometric index, due to its ability to estimate both total adiposity and fat distribution by using very simple measurements ${ }^{(13)}$ and its correlation with major clinical outcomes ${ }^{(14)}$.

A major limitation of the anthropometry-based measures to estimate \%BF is the limited generalisability of the equations to sub-populations whose body composition is very different from the population average ${ }^{(15)}$. Therefore, a great body of research in recent decades has been dedicated to validating and modifying the conventional \%BF equations for children, people with chronic disease, non-European ethnic groups, elderly and athletes $^{(16,17)}$. Another limitation is disagreement on the exact way of measuring waist circumference ${ }^{(18,19)}$, despite being probably the most frequently taken anthropometric measure.

Abbreviations: \%BF, percentage body fat; DEXA, dual-energy X-ray absorptiometry.

* Corresponding author: H. Schermann, fax +972 3737 7002, email sheralmi@bu.edu 
In addition, an insufficient number of studies investigated the neck circumference, which nevertheless appears to be a good predictor of metabolic and cardiovascular morbidity ${ }^{(20,21)}$. In our study, we attempted to develop a new predictive equation that can be applied for the general population to estimate $\% \mathrm{BF}$. To validate our results, we have used DEXA measurements as a 'gold standard', allowing also for comparison of the results to other studies.

\section{Methods}

\section{Study population}

The study consisted of retrospectively reviewing the files of 700 men and women aged 20-60 years (men:women $=57 \%: 43 \%$ ) who registered to a health club. The demographic and anthropometric characteristics of this cohort are presented in Table 1. Subjects with poorly stabilised chronic medical conditions who could not be accepted to the health club were excluded from the cohort. The data obtained anonymously from the files included the following: age, sex, weight, height, skinfolds thickness at four sites (suprailiac, biceps, triceps and subscapular), neck circumference and abdominal circumference at the umbilical level. All the subjects also had their body composition measured by DEXA (Lunar; General Electric). A single experienced physical fitness instructor (Y. L.) took all measurements in the morning hours. The institutional review board approved the use of the above secondary data without informed consent (study no. 4195-17).

Age was recorded to the nearest year, weight $(\mathrm{kg})$ was recorded on a digital scale (SECA model 400; Seca North America) while the subject was dressed in shorts (and bra for females), height was measured (in $\mathrm{cm}$ ) by a wall mounted measuring tape (SECA model 206; Seca North America), and BMI was calculated accordingly. Skinfolds were measured using a digital caliper (Skyndex, LLC) according to the American College of Sports Medicine guidelines ${ }^{(22)}$ and $\% \mathrm{BF}_{\mathrm{SF}}$ was calculated by the Durnin-Womersley formulas for men and women ${ }^{(10)}$. Neck and abdomen circumferences were measured (in $\mathrm{cm}$ ) with a flexible tape at the levels of laryngeal prominence and umbilicus, respectively.

\section{Development of the new equation}

The files of two groups, consisting of 476 (68\%) and 224 (32\%) subjects had been reviewed. Each group consisted of all subjects who registered to the health club during two consecutive periods of time, without exclusion. The first group was used for the development of the predictive equation ('development' group) and the second group was the 'validation' groups. The general characteristics (age, weight, height, BMI) of the 'development' and 'validation' groups were compared using $t$ tests. All descriptive data are presented as means, standard deviations and ranges (Table 1). The differences between the groups (age, weight, $\mathrm{BMI}$ and $\% \mathrm{BF}_{\mathrm{SF}}$ ) were statistically significant but not substantial from the clinical perspective, and did not interfere with validation of the equation.

The first step towards constructing the final regression model was finding the univariate linear correlation between each measurement (or an existing equation) and the 'gold standard' DEXA measurement. Each correlation was examined visually using the Scatter-gram graph for any linear and non-monotonic relationships. Residuals were analysed to test for accuracy of the prediction models. The Durbin-Watson statistics was used to test for the presence of autocorrelation along each measurement scale and the variance inflation factor was used to check multicollinearity. Next, from the measures that met the criterion of simple usage in a field condition, those having the highest correlation coefficients were chosen. For example, the seven-site skinfold equation by Jackson and Pollock yielded good results but did not meet the requirement of feasibility measuring under field condition. As this did not meet the researchers' desire to present simple measurement and formulation for implementation, it was not included in further analysis. Sex was included, and two regressions were obtained. The model was adjusted for age group by adding it in the regression. To ensure that the findings were not accidental, the 'development' file was randomly split into two halves and tested by using the original formula. The gaps were minimal and the formulas were, therefore, processed for testing in the 'validation' set of data without any prior confirmatory analysis. Finally, as 'age' did not contribute significantly to the prediction of DEXA value it was omitted from the final equation.

\section{Validation of the new equation}

The equations that were developed have been validated in the 'validation' group ( $n$ 224). The predictive ability of the suggested equation was assessed as agreement with the 'gold standard' measurement of body fat by DEXA. The agreement between DEXA and $\% \mathrm{BF}_{\mathrm{cal}}$ was presented separately for men

Table 1. General characteristics of 'development' and 'validation' study populations (Mean values, standard deviations and ranges)

\begin{tabular}{|c|c|c|c|c|c|c|c|c|c|c|c|c|}
\hline & \multicolumn{6}{|c|}{ Male } & \multicolumn{6}{|c|}{ Female } \\
\hline & \multicolumn{3}{|c|}{ 'Development' ( $n$ 291) } & \multicolumn{3}{|c|}{ 'Validation' ( $n$ 111) } & \multicolumn{3}{|c|}{ 'Development' ( $n$ 185) } & \multicolumn{3}{|c|}{ 'Validation' (n 113) } \\
\hline & Mean & SD & Range & Mean & SD & Range & Mean & SD & Range & Mean & SD & Range \\
\hline Age (years) & $37 \cdot 1$ & $9 \cdot 8$ & $20-58$ & 38.7 & 9.8 & $21-60$ & $35 \cdot 3^{\star}$ & 8.9 & $20-57$ & $38 \cdot 3$ & $10 \cdot 5$ & $20-62$ \\
\hline Weight (kg) & $83 \cdot 8^{\star}$ & $14 \cdot 0$ & $52-131$ & 91.0 & $15 \cdot 8$ & $59-134$ & $64 \cdot 3^{\star}$ & $10 \cdot 3$ & $46-105$ & $70 \cdot 3$ & $13 \cdot 2$ & $48-119$ \\
\hline BMI $\left(\mathrm{kg} / \mathrm{m}^{2}\right)$ & $26 \cdot 6^{\star}$ & $4 \cdot 1$ & $16 \cdot 3-41 \cdot 5$ & 28.5 & 4.5 & $19 \cdot 4-43 \cdot 2$ & $23 \cdot 7^{\star}$ & 3.6 & $17 \cdot 2-41 \cdot 5$ & $25 \cdot 9$ & 4.5 & $14 \cdot 4-39 \cdot 3$ \\
\hline$\% \mathrm{BF} \dagger$ & $24 \cdot 2^{*}$ & 9.5 & $4 \cdot 5-46 \cdot 6$ & $28 \cdot 2$ & 8.6 & $5 \cdot 2-47 \cdot 5$ & $33.9^{*}$ & 8.4 & $13 \cdot 9-54.0$ & 38.5 & 8.6 & $16 \cdot 8-54.9$ \\
\hline
\end{tabular}

$\% \mathrm{BF}$, percentage body fat.

* Statistically significant between 'development' and 'testing' groups $(P<0.05)$.

$\dagger$ Calculated, using the four-skinfold equation by Durnin-Womersley. 
and women, using Bland-Altman plots, by the mean difference between the two measurements, and by the critical difference (half $95 \%$ CI of difference) ${ }^{(23)}$, and by Lin's measures of agreement (concordance correlation coefficient, accuracy and precision $)^{(24)}$. The accuracy of the equation was also expressed by the percentage of cases whose prediction fell within $\pm 5 \%$ of the DEXA value.

\section{Results}

The final equations to predict \%BF as measured by DEXA $\left(\% \mathrm{BF}_{\mathrm{cal}}\right)$ included height in $\mathrm{cm}(H)$, abdominal circumference in $\mathrm{cm}(A)$ and neck circumference in $\mathrm{cm}(N)$, and were as follows:

$$
\begin{aligned}
\% \mathrm{BF}_{\mathrm{cal}}(\text { men })= & 10 \cdot 111( \pm 6 \cdot 541)-0 \cdot 239( \pm 0 \cdot 035) H \\
& +0 \cdot 808( \pm 0 \cdot 029) A-0 \cdot 518( \pm 0 \cdot 128) \mathrm{N} \\
\% \mathrm{BF}_{\mathrm{cal}}(\text { women })= & 19 \cdot 197( \pm 7 \cdot 439)-0.239( \pm 0 \cdot 035) H \\
& +0 \cdot 808( \pm 0 \cdot 029) A-0.518( \pm 0 \cdot 128) \mathrm{N}
\end{aligned}
$$

Adjusted $R^{2}$ of the models for women and men were 0.65 and $0 \cdot 78$, respectively. The final model, which served as the basis for the above equations had an $R^{2}$ of 0.78 and a standard error of the estimate of 4.63 (Table 2).

We incorporated the anthropometric measures of the 224 subjects from the 'validation' set into the new equations and evaluated the agreement of the calculated values with the corresponding DEXA values. Bland-Altman plots comparing DEXA to $\% \mathrm{BF}_{\mathrm{cal}}$ in men and women are presented in Fig. 1(a) and (b), respectively. The central line demonstrates that there was no significant deviation from $0(P<0.05)$, indicating no fixed bias. Limits of agreement that contained $95 \%$ of the $\% \mathrm{BF}_{\mathrm{cal}}$ estimates were $\pm 7 \cdot 83 \%$ for men and $\pm 8.42 \%$ for women. The Lin concordance coefficient of $\%$ $\mathrm{BF}_{\mathrm{cal}}$ and DEXA was 0.89 in men and 0.86 in women. The accuracy of \%BF was 1.0 in both men and women, and precision was 0.9 and 0.88 , respectively (Table 3 ). Accuracy of $\% \mathrm{BF}_{\mathrm{cal}}$ was also presented as percentage of predictions falling within $5 \%$ of the corresponding DEXA value. The suggested equation predicted accurately $\% \mathrm{BF}$ in $79.5 \%$ of subjects. The remaining $20.5 \%$ were symmetrically distributed: $9.3 \%$ underestimations and $11.2 \%$

\begin{tabular}{|c|c|c|c|c|c|}
\hline Models & Parameter & $\beta$ & SE & Adjusted $R^{2}$ & SEE \\
\hline \multirow[t]{4}{*}{ Female-only model } & (Constant) & $31 \cdot 142$ & $11 \cdot 199$ & 0.65 & $4 \cdot 85$ \\
\hline & Height & -0.229 & 0.066 & & \\
\hline & Abdomen circumference & 0.938 & 0.054 & & \\
\hline & Neck circumference & $-1 \cdot 128$ & 0.267 & & \\
\hline \multirow[t]{4}{*}{ Male-only model } & (Constant) & 4.089 & 7.496 & 0.78 & $4 \cdot 66$ \\
\hline & Height & -0.235 & 0.039 & & \\
\hline & Abdomen circumference & 0.806 & 0.034 & & \\
\hline & Neck circumference & -0.274 & 0.143 & & \\
\hline \multirow{5}{*}{ Final equation } & (Constant) & $10 \cdot 111$ & 6.541 & 0.79 & 4.63 \\
\hline & Sex (0 if male, 1 if female) & 9.086 & 0.898 & & \\
\hline & Height & -0.239 & 0.034 & & \\
\hline & Abdomen circumference & 0.808 & 0.029 & & \\
\hline & Neck circumference & -0.518 & 0.128 & & \\
\hline
\end{tabular}
overestimations (Table 3, Fig. 2). Overall, the equations appear to

Table 2. Final equations variables in the 'validation' set ( $\beta$-Coefficients with their standard errors; adjusted $R^{2}$ and standard error of the estimates (SEE))

(a)

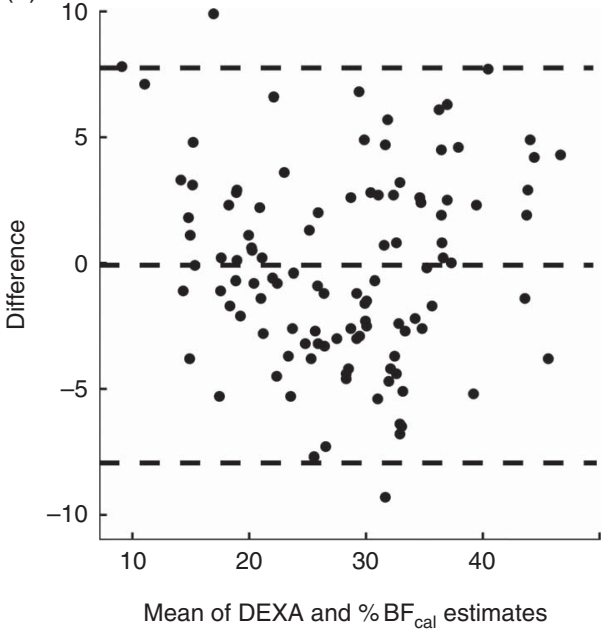

(b)

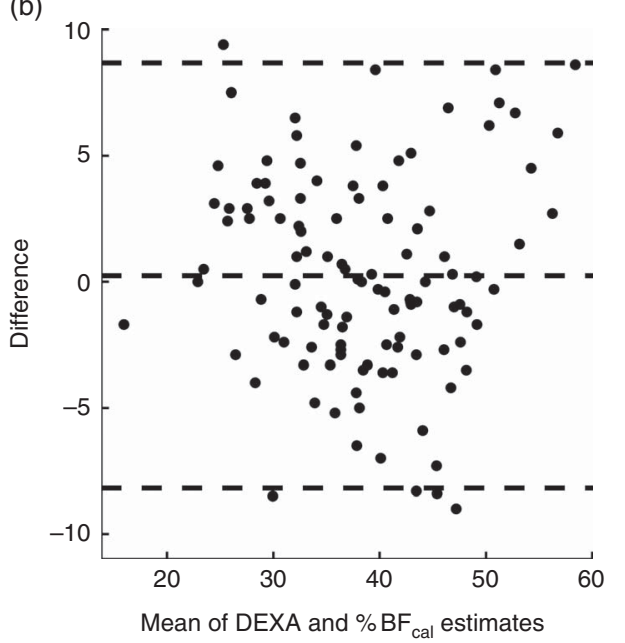

Fig. 1. Bland-Altman plots of agreement between the new anthropometric equation and the 'gold standard' body fat percentage (\%BF) estimate by dual-energy X-ray absorptiometry (DEXA) in males (a) and females (b). 
Table 3. Comparison of predictive ability of four skinfold formula $\left(\% \mathrm{BF}_{\mathrm{SF}}\right)$ and the new formula $\left(\% \mathrm{BF}_{\text {cal }}\right)$

\begin{tabular}{|c|c|c|c|c|}
\hline & \multicolumn{2}{|c|}{ Male } & \multicolumn{2}{|c|}{ Female } \\
\hline & $\% \mathrm{BF}_{\text {cal }}$ & $\% \mathrm{BF}_{\mathrm{SF}}$ & $\% \mathrm{BF}_{\text {cal }}$ & $\% \mathrm{BF}_{\mathrm{SF}}$ \\
\hline Bland-Altman mean difference from DEXA (\%) & -0.93 & $-2 \cdot 48$ & 0.24 & -3.01 \\
\hline Critical difference (half $95 \% \mathrm{Cl}$ ) & 7.83 & 8.96 & 8.42 & 7.9 \\
\hline Lin's concordance coefficient & 0.89 & 0.77 & 0.86 & 0.79 \\
\hline Lin's accuracy & 1 & 0.93 & 1 & 0.9 \\
\hline Lin's precision & 0.9 & 0.83 & 0.88 & 0.89 \\
\hline Percentage cases within $\pm 5 \%$ from DEXA prediction & 81 & 70 & 77 & 71 \\
\hline
\end{tabular}

\%BF, percentage body fat; DEXA, dual-energy X-ray absorptiometry.

(a)

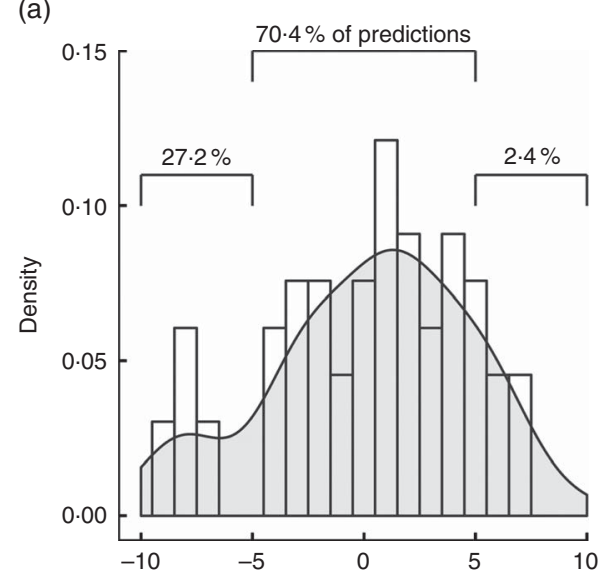

(b)

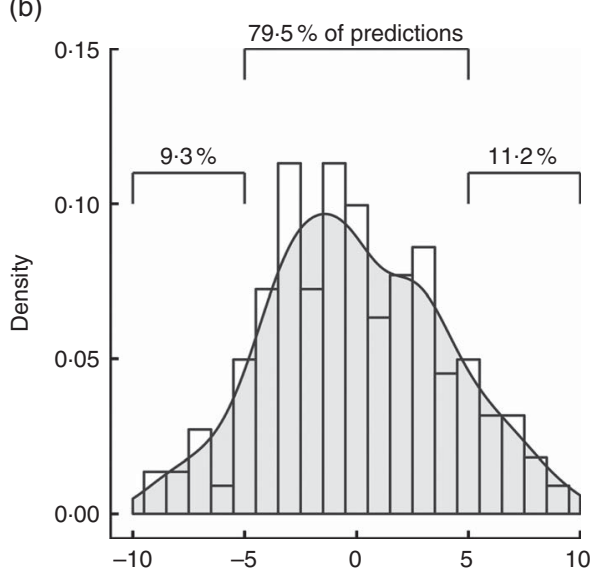

Fig. 2. Accuracy of prediction of percentage body fat (\%BF) by skinfolds equation (a) and the circumferences equation (b), represented as percentage of predictions falling within $5 \%$ of the dual-energy $\mathrm{X}$-ray absorptiometry value.

be externally valid for a large spectrum of measures (ages: 20-58 years, BMI: $14 \cdot 4-43 \cdot 2 \mathrm{~kg} / \mathrm{m}^{2}$, Table 1 ).

\section{Discussion}

The present study was undertaken to develop a simple but accurate predictive model to assess \%BF. Our final equation that consisted of height, waist circumference and neck circumference was proved to be valid, and can be applied for calculation of $\% \mathrm{BF}$ in the general population of men and women, ages $20-60$ years. This predictive equation is extremely useful for field applications due to the simplicity of measurements that it is based on.

Measuring body fat has multiple applications in various health and disease states. Although the direct measurement is impractical and indirect estimates usually employ sophisticated techniques, a great body of research addresses the doubleindirect estimates of body adiposity - equations that translate simple anthropometric measurements into \%BF. However, most of these measures, including BMI and waist:hip ratio (WHR), though widely used, correlate poorly with \%BF and clinical outcomes $^{(25)}$. We have observed similar discordance between BMI, WHR and DEXA also in our study (data not presented).

Equations for the assessment of body fat based on skinfold thickness measurements were developed in the $1950 \mathrm{~s}^{(8-10)}$. They have become a widely used tool as then, despite their relative complexity and questionable repeatability of the measurement technique. Noteworthy, previous studies indicate that measurement of body circumference is less prone to bias than skinfold caliper measurements ${ }^{(11,26)}$. In addition, recent research has shown the association of neck circumference with indirect measures of adiposity ${ }^{(27)}$ and with major clinical outcomes ${ }^{(20,21,28,29)}$. Thus, a great advantage of the newly developed model is the simplicity of measurements. While the models to assess \%BF by skinfold thickness provide a better estimate than BMI and WHR the method is challenging as it requires double or triple measurements at each anatomic location taken by a trained and experienced individual. Furthermore, the various models require complex calculations, and reliability among diverse ethnic groups is low ${ }^{(17,30)}$. To note, the skinfold equations were shown to become better predictors of $\% \mathrm{BF}$ after addition of waist and hip circumferences ${ }^{(17)}$.

Accuracy and precision of the new equation are within the range expected from anthropometric measures, according to values reported by other studies ${ }^{(31)}$. The Durnin-Womersley equation is a popular estimate of $\% \mathrm{BF}$ based on four skinfold measurements. We have used this estimate $\left(\% \mathrm{BF}_{\mathrm{SF}}\right)$ in the validation data set to calculate parameters of agreement with DEXA and compare them with those of the $\% \mathrm{BF}_{\text {cal }}$ equation. Bland-Altman plots demonstrated fixed bias, or underestimation of $\% \mathrm{BF}_{\mathrm{SF}}$ (by -2.48 and $-3.01 \%$ in men and women, respectively). This led to asymmetry in prediction of $\% \mathrm{BF}$, with about $27.2 \%$ of the subjects having underestimated \%BF determined by skinfolds, as opposed to only $9.3 \%$ by $\% \mathrm{BF}_{\text {cal }}$ (Fig. 2). In clinical use, one would opt for overestimation over 
underestimation of \%BF, as the latter implies 'missing' patients that could benefit from lifestyle interventions. It follows that in order to accurately predict \%BF by the four-skinfolds method, adjustment is required (a correction factor of 1.1 for men and 1.03 for women was calculated for the present set of data). Currently, adding an adjusting coefficient is not the common practice that may add complexity to the already complicated method. Lin's concordance coefficient, accuracy and precision of $\% \mathrm{BF}_{\mathrm{cal}}$ were higher than for $\% \mathrm{BF}_{\mathrm{SF}}$. Although $79.5 \%$ of the new predictive model were within $\pm 5 \%$ from the values obtained by DEXA, only $70.4 \%$ of $\% \mathrm{BF}_{\mathrm{SF}}$ were within $\pm 5 \%$ of the 'gold standard' (Fig. 2). Even after the above-mentioned adjustments $\% \mathrm{BF}_{\mathrm{SF}}$ was less precise than $\% \mathrm{BF}_{\text {cal }}$ in estimating correctly $\% \mathrm{BF}$ and only $72 \%$ of the results of men and $76 \%$ of the results in women were within $\pm 5 \%$ of the DEXA values.

There are some limitations to the study, to which the potential user of the proposed equations should be aware. In the study, we used DEXA as a 'gold standard'. Despite being accepted as the method of choice, it remains an indirect measure of body composition and its results are influenced by technique of testing and by the subjects' physiological status (e.g. level of hydration). In part this was overcome by taking all measurements in the morning hours. Although we have tried to include a large heterogeneous cohort in our study, the equations were developed using a database of a group of healthy Israeli adults of relatively high socioeconomic background, which is implied by their ability to afford visiting a health club. Nevertheless, the validity of the equations appears to be externally valid for a large spectrum of measures (ages: $20-58$ years, BMI: $14.4-43.2 \mathrm{~kg} / \mathrm{m}^{2}$, Table 1). Yet, the equations presented here require further validation of the formula in sub-populations, such as soldiers, elderly, children and bedridden patients, in whom the use of field measures of adiposity is of importance. The latter may be the greatest beneficiaries from the formula, as it does not require weighing the person to predict $\% \mathrm{BF}$.

To summarise, in the community, athletic trainers and nutritionists can effectively use the suggested model due to its simplicity and applicability. The three variables that are used make the model advantageous over all other models, with no need for an expensive device or particular experience.

\section{Acknowledgements}

This research received no specific grant from any funding agency, commercial or not-for profit sectors.

All authors contributed to study design, conduct, data collection and analysis, writing and critical review of the final manuscript.

The authors declare that there are no conflicts of interest.

\section{References}

1. Simões M, Severo M, Oliveira A, et al. (2016) Predictive equations for estimating regional body composition: a validation study using DXA as criterion and associations with cardiometabolic risk factors. Ann Hum Biol 43, 219-228.

2. Francis KT (1990) Body-composition assessment using underwater weighing techniques. Phys Ther 70, 657-662.

3. Nana A, Slater GJ, Stewart AD, et al. (2015) Methodology review: using dual-energy X-ray absorptiometry (DXA) for the assessment of body composition in athletes and active people. Int J Sport Nutr Exerc Metab 25, 198-215.

4. Sheng HP, Adolph AL, Smith EO, et al. (1988) Body volume and fat-free mass determinations by acoustic plethysmography. Pediatr Res 24, 85-89.

5. Cornier MA, Després JP, Davis N, et al. (2011) Assessing adiposity: a scientific statement from the American Heart Association. Circulation 124, 1996-2019.

6. Batsis JA, Mackenzie TA, Bartels SJ, et al. (2016) Diagnostic accuracy of body mass index to identify obesity in older adults: NHANES 1999-2004. Int J Obes 40, 761-767.

7. Pasco JA, Nicholson GC, Brennan SL, et al. (2012) Prevalence of obesity and the relationship between the body mass index and body fat: cross-sectional, population-based data. PLOS ONE 7, e29580.

8. Siri WE (1956) The gross composition of the body. Adv Biol Med Phys 4, 239-280.

9. Jackson AS \& Pollock ML (2004) Generalized equations for predicting body density of men. 1978. Br J Nutr 91, 161-168.

10. Durnin JV \& Womersley J (1974) Body fat assessed from total body density and its estimation from skinfold thickness: measurements on 481 men and women aged from 16 to 72 years. Br J Nutr 32, 77-97.

11. Kannel WB, Cupples LA, Ramaswami R, et al. (1991) Regional obesity and risk of cardiovascular disease; the Framingham Study. J Clin Epidemiol 44, 183-190.

12. Mueller WH \& Malina RM (1987) Relative reliability of circumferences and skinfolds as measures of body fat distribution. Am J Phys Anthropol 72, 437-439.

13. Pietrobelli A \& Brambilla P (2014) Body composition measurements. Ital J Pediatr 40, Suppl. 1, A6.

14. Savva S, Lamnisos D \& Kafatos A (2013) Predicting cardiometabolic risk: waist-to-height ratio or BMI. A meta-analysis. Diabetes Metab Syndr Obes 6, 403-419.

15. Going S, Lee V, Blew R, et al. (2014) Top 10 research questions related to body composition. Res Q Exerc Sport $\mathbf{8 5}$, $38-48$.

16. Hronek M, Kovarik M, Aimova P, et al. (2013) Skinfold anthropometry - the accurate method for fat free mass measurement in COPD. COPD 10, 597-603.

17. Kohli S, Gao M \& Lear SA (2009) Using simple anthropometric measures to predict body fat in South Asians. Appl Physiol Nutr Metab 34, 40-48.

18. Ma W-Y, Yang C-Y, Shih S-R, et al. (2013) Measurement of waist circumference: midabdominal or iliac crest? Diabetes Care 36, 1660-1666.

19. World Health Organization (2008) Waist Circumference and Waist-Hip Ratio. Report of a WHO Expert Consultation. Geneva: WHO.

20. Preis SR, Massaro JM, Hoffmann U, et al. (2010) Neck circumference as a novel measure of cardiometabolic risk: the Framingham Heart Study. J Clin Endocrinol Metab 95, 3701-3710.

21. Vallianou NG, Evangelopoulos AA, Bountziouka V, et al. (2013) Neck circumference is correlated with triglycerides and inversely related with HDL cholesterol beyond BMI and waist circumference. Diabetes Metab Res Rev 29, 90-97.

22. Ratamess N (2013) Body composition status and assessment. In ACSM's Guidelines for Exercise Testing and Prescription, 9th ed., pp. 264-281 [JK Ehrman, editor]. Philadelphia, PA: LWW.

23. Bland JM \& Altman DG (1986) Statistical methods for assessing agreement between two methods of clinical measurement. Lancet i, 307-310.

24. Lin L, Hedayat AS, Sinha B, et al. (2002) Statistical methods in assessing agreement: models, issues, and tools. I Am Stat Assoc 97, 257-270. 
25. Navaneethan SD, Kirwan JP, Arrigain S, et al. (2014) Adiposity measures, lean body mass, physical activity and mortality: NHANES 1999-2004. BMC Nephrol 15, 108.

26. Himes JH, Roche AF \& Siervogel RM (1979) Compressibility of skinfolds and the measurement of subcutaneous fatness. Am J Clin Nutr 32, 1734-1740.

27. Yang L, Samarasinghe Y, Kane P, et al. (2009) Visceral adiposity is closely correlated with neck circumference and represents a significant indicator of insulin resistance in WHO grade III obesity. Clin Endocrinol 73, 197-200.

28. Luo Y, Ma X, Shen Y, et al. (2017) Neck circumference as an effective measure for identifying cardio-metabolic syndrome: a comparison with waist circumference. Endocrine $\mathbf{5 5}$, 822-830.

29. Huang BX, Zhu MF, Wu T, et al. (2015) Neck circumference, along with other anthropometric indices, has an independent and additional contribution in predicting fatty liver disease. PLOS ONE 10, e0118071.

30. Davidson LE, Wang J, Thornton JC, et al. (2011) Predicting fat percent by skinfolds in racial groups: Durnin and Womersley revisited. Med Sci Sports Exerc 43, 542-549.

31. Peterson CM, Thomas DM, Blackburn GL, et al. (2016) Universal equation for estimating ideal body weight and body weight at any BMI. Am J Clin Nutr 103, 1197-1203. 